\title{
CASE REPORT: CONCEALED SUBACUTE LEFT VENTRICULAR FREE WALL RUPTURE POST-MYOCARDIAL INFARCTION: ANOTHER DIAGNOSTIC DILEMMA
}

\author{
TS Banerjee, Medical Student; HT Tan, SpR in Gastroenterology; \\ SE Penney, HO; JJ Keating, Consultant Physician \\ Furness General Hospital
}

Left ventricular wall rupture is a well known, fatal complication of myocardial infarction and accounts for a large $10 \%$ of infarct-related death ${ }^{(1,2)}$. Its subacute form is potentially salvageable and occurs in $30 \% \%^{(2)}$. It usually presents with a sudden haemodynamic collapse, tamponade, and electromechanical dissociation ${ }^{(2)}$.

\section{CASE REPORT}

A 75-year old lady was admitted to Furness General Hospital at 0830 hours with shortness of breath. She had become increasingly breathless over the preceding week despite being prescribed antibiotics by her GP for a presumed chest infection. She denied any chest pain, her only other symptom being a non-productive dry cough.

Past medical history revealed mild hypertension and rheumatoid arthritis which were being treated with atenolol and methotrexate respectively. She was a non-smoker and kept no pets or birds.

On examination she was noted to be tachypnoeic and tachycardic. She was apyrexial with a blood pressure of 118/76. Cardiovascular examination revealed clear heart sounds, a normal jugular venous pressure and no ankle oedema. Fine bilateral crepitations were heard on auscultation in the lung bases and mid-zones. Abdominal and neurological examinations were unremarkable.

Clinical investigations revealed leucocytosis with neutrophilia. The white cell count was $12 \times 10^{9} / \mathrm{L}$. Arterial blood gases showed Type I respiratory failure with compensatory alkalosis $\mathrm{PO}_{2} 5.5 \mathrm{kPa}, \mathrm{PCO}_{2} 3.3 \mathrm{kPa}, \mathrm{pH} 7.5$, base excess -1.9 . Her chest X-ray revealed a large globular heart with pulmonary oedema. Her electrocardiogram (ECG) showed a right bundle branch block with sinus tachycardia and normal voltage. Cardiac enzymes were normal.

A working diagnosis of acute congestive cardiac failure was made, possibly with a superimposed pneumonia. Methotrexate pneumonitis was a differential diagnosis. Despite treatment with intravenous diuretics and antibiotics, $100 \%$ oxygen and chest physiotherapy, she remained significantly hypoxic. She also experienced recurrent drops in oxygen saturation followed by a temporary drop in blood pressure.
At 1800 hours, the patient was noted to be oliguric and her central venous pressure was $9 \mathrm{~cm} \mathrm{H}_{2} \mathrm{O}$. By 2200 hours the following day, the patient had deteriorated significantly. She developed a temperature of $37.7^{\circ} \mathrm{C}$ and was noted to be agitated. Her central venous pressure had fallen to $-2 \mathrm{~cm}$ of water and oxygen saturation was $60 \%$ on $100 \%$ oxygen. Blood pressure was $95 / 61$. She was transferred to the Intensive Care Unit to be intubated and ventilated.

An adrenaline infusion was commenced in order to support the patient's failing cardiovascular system. Arterial blood gases on an $\mathrm{Fi} \mathrm{O}_{2}$ of $78 \%$ showed a much improved oxygenation of $\mathrm{PO}_{2} 21.4 \mathrm{kPa}$ but she had developed a metabolic acidosis as shown by a $\mathrm{pH}$ of 7.25 and base excess of -13.8 .

At 1000 hours the patient remained markedly acidotic and her adrenaline requirements were increasing. Sodium bicarbonate did not appear to improve the acidosis and haemofiltration was commenced. By 1030 hours she was noted to have an elevated glucose of $20.2 \mathrm{mmol} / \mathrm{L}$ and an insulin infusion was started. Her adrenaline requirements continued to increase and it was becoming difficult to keep her blood pressure above 50/40.

Unfortunately, the patient had an electromechanical dissociation cardiac arrest at 1600 on day three. Cardiopulmonary resuscitation was unsuccessful. A pericardiocentesis was performed and blood was withdrawn from the pericardial space but the patient failed to respond.

A post-mortem examination revealed a pericardial haemorrhage secondary to a rupture in the antero-septal ventricular wall at the site of an acute myocardial infarction thought to have taken place some 10 days previously. There was also significant arteriosclerosis in the left descending artery, circumflex and right coronary artery.

\section{DISCUSSION}

Left ventricular free wall rupture is an unpredictable event which usually leads to death. It is regarded as the third major complication and second most common cause of death following myocardial infarction ${ }^{(3)}$. Despite its frequency, diagnosis is difficult because there is no typical presentation. 
Ventricular free wall rupture can be acute or subacute. The former involves an abrupt rupture of the infarcted area resulting in haemopericardium and death within 30 minutes $^{(4)}$. A subacute form, however, results in slow or repetitive bleeding in the pericardial sac due to gradual or incomplete rupture of the infarcted area. Without surgery, complete terminal rupture can occur from 30 minutes to a few days following the initial clinical event ${ }^{(4)}$

Various studies have attempted to form criteria to diagnose left ventricular wall rupture. Figueras et al depended on the sudden development of electromechanical dissociation without preceding heart failure with bradycardia, acutely distended neck veins and a cyanosed head and neck ${ }^{(5)}$. Twelve and a half percent of these patients developed an acute nonarrythmic hypotension (systolic blood pressure $<80 \mathrm{mmHg})^{(5)}$. Oliva et al found that $95 \%$ of patients suffered from two or three symptoms of positional pleuritic pain, vomiting and restlessness with agitation ${ }^{(6)}$. Purcaro et al made diagnoses of ventricular wall rupture based upon haemodynamic deterioration during the acute infarct, pericardial effusion, intrapericardial echoes suggesting clots or extraction of blood by pericardiocentesis, cardiac tamponade, and visualisation of the rupture by echocardiography ${ }^{(4)}$.

The variety of signs and symptoms within these different studies highlight the difficulty in diagnosis. There does not appear to be a typical pattern which can be routinely applied to presenting patients. Clinical investigations indicative of ventricular wall rupture have been shown to include ECG abnormalities. Oliva et al concluded that persistent ST elevation in addition to the clinical symptoms was a good predictor for rupture ${ }^{(6)}$. Khogali et al also stated that ECG changes in left ventricular rupture, though not marked, are usually abnormal ${ }^{(1)}$. Chest radiographs did not appear to be of relevance to diagnosis. However, with regard to echocardiography, several studies and case reports have documented its invaluable aid. Such non-invasive imaging has allowed early diagnosis and ultimately increased attempts at lifesaving surgical treatment ${ }^{(3,7)}$.

Risk factors for ventricular free wall rupture include older age, the female gender, hypertension and the absence of previous myocardial infarction ${ }^{(7)}$. Thirty-two percent of ruptures occur within 24 hours of an infarction while most occur within the first week $^{(8,7)}$. The use of $\beta$-blockers can help to reduce the incidence of left ventricular free wall rupture by lowering blood pressure and decreasing myocardial contractility ${ }^{(5)}$. Our patient was being treated with atenolol perhaps explaining delayed rupture. An American study suggested that patients who underwent thrombolytic therapy within the first 24 hours following infarction had a significantly reduced incidence of rupture on or after day four ${ }^{(7)}$. There may, however, be evidence that such therapy can actually increase the incidence of early cardiac rupture ${ }^{(9)}$.

This case is unusual in that there was no evidence of any chest pain. Whilst the classical presentation of a myocardial infarction involves chest pain at rest, lasting for several hours coupled with restlessness, up to $20 \%$ of the population experience silent myocardial infarctions which go unnoticed $^{(10)}$, especially in diabetics. The only possible indications of such an event in our case were complications of sinus tachycardia and right bundle branch block. The latter occurs in $20-30 \%$ with progressive AV block and ultimate QRS axis shift ${ }^{(10)}$. The patient did indeed suffer from previously undetected diabetes. Had this been known, the risk of a silent myocardial infarction would have been more apparent, consequently raising the possibility of rupture. Recurrent hypotension and hypoxia were the only signs consistent with rupture of the ventricular wall. This could perhaps have been an indication for echocardiography since hypotension is considered to be a sensitive marker of subacute ventricular free wall rupture and can present acutely or progressively (although associated with tamponade) ${ }^{(1,5)}$. Cardiac tamponade was not detected. There was no evidence of arterial pulsus paradoxus and heart sounds were clear. Hypotension and a globular shaped heart on chest X-ray could have been suggestive. Haemodynamic signs of cardiac tamponade may have been absent due to a very slow intrapericardial bleed ${ }^{(4)}$. This may also occur due to an improvement or a reversal of tamponade should the leakage temporarily subside ${ }^{(4)}$. Such an occurrence could be due to a thrombus overlying the site of rupture, providing temporary protection ${ }^{(5)}$. Cases such as these have previously been identified and can be described as a type III rupture ${ }^{(7,3)}$. In such cases, intrapericardial echoes can prove invaluable ${ }^{(4)}$ and this may indeed have been relevant in this case.

Differential diagnoses included methotrexate pneumonitis or a pulmonary embolus. The former involves pulmonary toxicity shown by dyspnoea and cough, which did affect the patient. The latter was suggested by arterial blood gases and ECG, though not by her chest X-ray. Tinzaparin was administered without response. Possible atypical pneumonia was treated with antibiotics. Cardiac enzyme measurements proved unhelpful. It may have been useful to perform a Troponin $\mathrm{T}$ measurement since this remains elevated following normalisation of creatine kinase levels.

In conclusion, this patient proved the difficulty of diagnosing post myocardial infarction left ventricular free wall rupture. There was no evidence of an infarction and the rupture occurred outside the normal bracket of one week, thereby making the correct diagnosis highly difficult.

This case highlights two important points. The first shows the need for a high index of suspicion in situations involving unexplained heart failure and recurrent hypotension which do not respond to usual treatment. Access to emergency echocardiography can prove invaluable in confirming the diagnosis. Secondly, there is a need for increased awareness of patients at risk. The patient's risk factors proved more suggestive of potential rupture than her clinical signs. The significance of untreated diabetes leading to the diagnosis of a silent myocardial infarction should also be emphasised. Thus, in the face of so many contradicting clinical signs, these are currently the best ways to confront this challenging problem and avoid needless deaths.

\section{REFERENCES}

1 Khogali SS, Bonser RS, Beattie JM Concealed post-infarction left ventricular rupture - a diagnostic dilemma Postgrad Med J 1996;72(844):121-122

2 Tate DA, Lawton JL, DeGent G, Smith SC Jnr Subacute ventricular free wall rupture presenting as tamponade without frank hemopericardium Catheterisation and Cardiovascular Diagnosis 1998;44:417-419

3 Reardon MJ, Carr CL, Diamond A, Letsou GV, Safi HJ, Espada R, Baldwin JC Ischemic left ventricular free wall rupture: prediction, diagnosis, and treatment Am Thorac Surg 1997;64:1509-1513 
4 Purcaro A, Costantini C, Ciampani N, Mazzanti M, Silenzi C, Gili A, Belardinelli R, Astolfi D Diagnostic criteria and management of subacute ventricular free wall rupture complicating acute myocardial infarction Am J Cardiol 1997;80:397-405

5 Figueras J, Cortadellas J, Evangelista A, Soler-Soler J Medical management of selected patients with left ventricular free wall rupture during acute myocardial infarction JACC 1997;29:512-518

6 Oliva PB, Hammil SC, Edwards WD Cardiac rupture, a clinically predictable complication of acute myocardial infarction: report of 70 cases with clinicopathologic correlations J Am Coll Cardiol 1993;22:720-726
7 Sutherland, FWH, Guell FJ, Pathi VL, Naik SK Postinfarction ventricular free wall rupture: strategies for diagnosis and treatment Ann Thorac Surg 1996;61:1281-1285

8 Zahger D, Milgalter E Clinical problem solving: a broken heart N Engl J Med 1996;334:319-321

9 Becker RC, Gore JM, Lambrew C, Weaver WD, Rubison RM, French WJ, Tiefenbrunn AJ, Bowlby RN, Rogers WJ A composite view of cardiac rupture in the united states national registry of myocardial infarction JACC 1996;27:1321-1326

10 Kumar PJ, Clark ML Clinical Medicine 3rd edition WB Saunders Company Ltd, London: 1994:583-588 